\title{
Weight Status and Dietary and Physical Activity Behavior Differences between Hispanic and Non-Hispanic Children in the United States
}

\author{
Beth L. Ellcessor ${ }^{\mathrm{a}}$ and Amber R. Haroldson ${ }^{\mathrm{a}}$
}

\begin{abstract}
Background: Hispanic children have a higher prevalence of overweight and obesity in the United States. Acculturation of Hispanics living in the U.S. affects dietary intake and physical activity, thus impacting weight status and health problems related to obesity.

Purpose: The purpose of this study was to examine the differences in overweight/obesity and dietary and physical activity behaviors between Hispanic and non-Hispanic children living in the United States.

Methods: Using the 2001-2002 Health Behaviors of School-Aged Children Questionnaire, $6^{\text {th }}-10^{\text {th }}$ grade student surveys $(n=14,817)$ were analyzed to compare BMI, consumption of fruits, vegetables, and sugar-sweetened beverages, and levels of physical activity between Hispanic and non-Hispanic subjects. Independent t-test, Oneway ANOVA and Pearson's Chi-Square provided statistical analysis for the descriptive and research variables.

Results: Comparing Hispanic and non-Hispanic students, there were no differences in gender or age, but urbanicity, birth country, home language, and education of mother and father had significant differences between Hispanic and non-Hispanic students $(p<0.01)$. The Hispanic sample had higher rates of overweight and obesity $(p<0.001)$, consumed lower amounts of fruits $(\mathrm{p}<0.001)$ and vegetables $(\mathrm{p}<0.001)$, consumed higher amounts of sugar-sweetened beverages $(\mathrm{p}=0.001)$, and reported being less physically active ( $\mathrm{p}<0.001)$ compared to non-Hispanic students.

Conclusion: As evidenced by the 2001-2002 HBSC survey, Hispanic children are consuming less fruits and vegetables and more sugar-sweetened beverages, and have lower rates of physical activity compared to non-Hispanic children. This may be contributing to the higher rates of overweight and obesity found in this population. These results could be used in the development of childhood obesity interventions involving Hispanic children.
\end{abstract}

Keywords: Hispanic children; childhood obesity; dietary intake; physical activity

\section{Introduction}

To assess the incidence of obesity in school aged children, Cunningham, Kramer, and Narayan (2014) followed a representative sample of over 9000 students from kindergarten through eighth grade in the United States. The obesity prevalence rate for kindergarten children was $12.4 \%$ and increased to $20.8 \%$ by eighth grade. Hispanic children by eighth grade had a $26.6 \%$ prevalence rate of obesity, which is 5.8 percentage points greater than the overall prevalence rate and 9.6 percentage points greater than non-Hispanic white children of the same age (Cunningham et al., 2014).

According to NHANES 1999-2002, 21.8\% and $22.5 \%$ of Mexican American children were overweight or obese for 611 years old and 12-19 years old, respectively. For Mexican American boys 6-11 years old, the overweight or obese prevalence rate increased 9\% from the 1988-1994 to the 1999-2002 NHANES. Similarly, a 10.6\% increase occurred for the 12-19 year old boys of these same demographics in the same time frame (Baskin, Ard, Franklin, \& Allison, 2005). In 2011-2012, Hispanic children living in the United States had a $22.4 \%$ obesity prevalence rate, which is significantly higher than the $16.9 \%$ overall obesity prevalence rate for children in the United States (Ogden, Carroll, Kit, \& Flegal, 2014).

It is evident that Hispanic children in the United States have a higher prevalence rate of obesity than comparative non-Hispanic children. According to Daniels (2006) the longterm consequences of obesity in children lead to an increased risk of health problems. Among these are hypertension and atherosclerosis, which can lead to cardiovascular disease, dyslipidemia, type 2 diabetes, asthma and obstructive sleep apnea, nonalcoholic fatty liver disease, gastroesophageal reflux, and a disorder of the hip growth plate. In addition to physical health problems, mental health can also be affected. Depression is a psychosocial disorder of increased prevalence for obese children and adolescence.

In efforts to prevent negative health outcomes in Hispanic children, there is a need to investigate what factors may contribute to their increased prevalence rate of obesity. Many Hispanic or Latino Americans who have immigrated to the United States attest to eating more processed food, fast food, and snack food than they ate in their countries of origin, often leading to uncontrolled weight gain (Cason, NietoMontenegro, \& Chavez-Martinez, 2006; Greaney, Lees, Lynch, Sebelia, \& Greene, 2012). The change in diet, influenced by adjusting to a new culture, could be a main contributor to increased obesity among Hispanic children in the United States.

This adjustment to a new culture, known as acculturation, has been shown to impact Hispanics immigrants, where those who are more immersed in American culture, tend to have a lower quality of health and nutrition (Greaney et al., 2012). Dave, Evans, Saunders, Watkins, and Pfeiffer (2009) found that children from families who were less acculturated had higher fruit and vegetable intake than children from families who were more acculturated $(\mathrm{p}<0.001)$. Additionally, lower fruit and vegetable consumption among Hispanic children has been 
directly correlated with high acculturation levels and also low-income at home (Dave et al., 2009). Wiley et al. (2014) has also studied how acculturation of Puerto Rican or nonPuerto Rican Hispanic mothers and caregivers affects child BMI percentile. Non-core food consumption, such as sugarsweetened beverages, processed foods, and sweets, was found to be the major factor linking child BMI percentiles and acculturation in Puerto Ricans and non-Puerto Rican Hispanics (Wiley et al., 2014).

Food intake and poor nutrition are not the only factors contributing to obesity. Physical activity is another crucial component for maintaining a healthy lifestyle. Acculturation has also been shown to affect physical activity levels of Hispanic Americans. Liu, Probst, Harun, Bennett, and Torres (2009) examined the relationship between acculturation and physical activity. Foreign-born Hispanic children living in the United States were less likely to reach physical activity recommendations than native Hispanic children whose parent(s) or grandparents were born outside of the United States (2009, p. 517). While acculturation has not been shown to have a causative effect on obesity, it has been correlated with negative dietary intake patterns, such as decreased fruit and vegetables and increased sugar-sweetened beverages, sweets, and processed foods, as well as with decreased physical activity.

The purpose of this secondary data analysis was to examine the differences in overweight/obesity and dietary and physical activity behaviors between Hispanic and nonHispanic children living in the United States, using the 20012002 Health Behaviors of School-Aged Children Questionnaire. It was hypothesized that 1) Hispanic children would have a higher rate of overweight/obesity than nonHispanic children, 2) Hispanic children would have lower fruit and vegetable intake than non-Hispanic children, 3) Hispanic children would consume more sugar-sweetened beverages than non-Hispanic children, and 4) Hispanic children would have lower levels of physical activity than non-Hispanic children.

\section{Experimental Procedures}

This was a secondary data analysis study utilizing the Health Behavior in School-Aged Children (HBSC) 2001-2002 cross-sectional survey. The data was made available to the public in August 2005 and was retrieved online at http://www.hbsc.org. HBSC is a nationally representative survey of students in grades six through ten, and also includes data from school staff and administration. The purpose of the HBSC survey was to better understand the factors of health behavior in young people in order to influence health promotion, policy, and future interventions for this population. The HBSC was conducted in 35 countries with cooperation from the World Health Organization.

\section{Participants}

This analysis included only participants in the United States. The target population included children enrolled in public, Catholic, and other private schools from all 50 states. Public schools were recruited using Probability Proportional to Size (PPS) methodology to obtain a sample that represented the national population. Private schools were stratified by geographic location and selected as a single-stage sample due to overall smaller proportion of private schools. Classes within each selected school were chosen using simple random sampling, and all students within selected classes were asked to participate in the study.

The original sample size of schools was 548 and a total of 340 schools participated in the survey $(62.5 \%)$. The response rate of students within participating schools was $81.9 \%$, with a total of 15,245 school-aged students surveyed. Due to exclusion factors, such as responding students missing several key variables $(n=57)$, being outside of target age or grade population $(n=365)$, or having unknown age and grade $(n=6)$, the data represents 14,817 students. Students of minority ethnicity (i.e. Hispanic and African American) were over-sampled to provide estimates within $5 \%$ precision at a 90\% confidence interval. School administrators and health educators were also surveyed about health related factors within their respective schools, but these responses were not included in this analysis.

\section{Data Collection}

HBSC 2001-2002 surveyed students about nutrition, physical activity, violence, relationships, safety at school, substance use, and the community in which they live. The questionnaire included 77 multiple-choice questions with an additional 15 questions for high-school students only. The survey was a paper questionnaire given in class during the school day and estimated to take approximately 45 minutes to complete. Each survey was coded with an ID number to uphold anonymity and confidentiality.

\section{Variables}

The following variables were utilized in this analysis: Hispanic ethnicity, body mass index (BMI), fruit and vegetable consumption, sugar-sweetened beverage consumption, and physical activity. Identification of Hispanic participants was made utilizing one survey item: "Are you Hispanic or Latino?" The responses included "No, I am not Hispanic or Latino" or "Yes, I am Hispanic or Latino." Data with missing responses for this question were not used in the statistical analysis $(n=369)$.

BMI was computed based on the self-reported weight and height of each student. The following formula was used to calculate BMI: [Weight (lbs) / [Height (inches) * Height (inches)]] * 703. BMI-for-age, which takes into account the child's gender and age, was used to interpret the child's BMI. Age was imputed based on grade level, month and year of birth. The Centers for Disease Control and Prevention (CDC) 2000 growth charts were used to plot BMI-for-age and categorize weight status (U.S. Dept. of Health and Human Services, 2003). Four categories were included: "Underweight" was considered less than the $5^{\text {th }}$ percentile, "Healthy weight" was between the $5^{\text {th }}$ and $85^{\text {th }}$ percentile, "At risk for overweight" was between the $85^{\text {th }}$ and $95^{\text {th }}$ percentile, and "Overweight" was considered greater than the $95^{\text {th }}$ percentile. The definitions of overweight and obese have changed since the survey was conducted in 2001-2002. Currently, overweight is defined as between the $85^{\text {th }}$ and $95^{\text {th }}$ percentile and obese is defined as greater than the $95^{\text {th }}$ percentile.

Fruit and vegetable consumption was assessed using two questions: "How many times a week do you eat or drink fruits?" and "How many times a week do you eat or drink 
vegetables?" Possible responses were divided into seven categories: "Never," "Less than once a week," "Once a week," "2-4 days a week," "5-6 days a week," "Once a day, everyday," or "Everyday, more than once." For this analysis, the bottom three responses ("Never," "Less than once a week," and "Once a week") were grouped to create a category of low consumption. The top three responses ("5-6 days a week," "Once a day, everyday," and "Everyday, more than once") were grouped to create a category of high consumption.

Consumption of sugar-sweetened beverages was measured using one survey item: "How many times a week do you drink coke or other soft drinks that contain sugar?" Response options were the same as those of fruit and vegetable consumption, ranging from "Never" to "Everyday, more than once." Similar to the fruit and vegetable responses, the bottom three responses ("Never," "Less than once a week," and "Once a week") were grouped to create a category of low consumption. The top three responses ("5-6 days a week," "Once a day, everyday," and "Everyday, more than once") were grouped to create a category of high consumption.

Physical activity was defined and described by HBSC within each student survey.

Physical activity is any activity that increases your heart rate and makes you get out of breath some of the time. Physical activity can be done in sports, school activities, playing with friends, or walking to school. Some examples of physical activity are running, brisk walking, rollerblading, biking, dancing, skateboarding, swimming, soccer, basketball, football, and surfing (U.S. Dept. of Health and Human Services, 2003, p. 4).

Two questions assessed physical activity: "Over the past 7 days, on how many days were you physically active for a total of at least 60 minutes per day?" and "Over a typical or usual week, on how many days are you physically active for a total of at least 60 minutes per day?" Responses included one day increments, ranging from "0 days" to "7 days". For this analysis, the bottom three responses ( 0 days, 1 day, and 2 days) were grouped to create a category of low physical activity ( 2 days or less). The top three responses ( 5 days, 6 days, and 7 days) were grouped to create a category of high physical activity (5 days or more).

\section{Statistical Analysis}

All statistical tests were conducted using SPSS statistical analysis software (version 22, IBM Corporation). Frequency counts were run for sample demographic variables (i.e. gender, age, urbanicity, birth country, home language, and parent education). Oneway ANOVA and Pearson's ChiSquare $\left(X^{2}\right)$ were used to determine differences among the dependent variables (e.g. BMI or fruit consumption) by the independent variable (Hispanic or non-Hispanic). Dependent variables of continuous measure were tested using independent t-test or ANOVA and Levene's test for homogeneity of variances. Dependent variables of categorical measure were tested using $X^{2}$ and Phi and Cramer's V. Statistical significance was indicated with an alpha level of 0.05 ( $\mathrm{p}<0.05)$. Missing data was not included in this analysis.

\section{Results}

\section{Description of participants}

Demographic information of the total sample $(n=14,817)$ broken down by Hispanic $(\mathrm{n}=2,889)$ and non-Hispanic $(n=11,559)$ can be found in Table 1. Age ranged from 10-17 years old, with $54.9 \%$ of participants 13 years old or younger. The most common age was 12 years old $(20.6 \%)$ and the least common ages were 10 or 17 ( $0.2 \%$ and $0.6 \%$ respectively). For urbanicity, more students lived in an urban area, than suburban or rural areas. Well over the majority (92.6\%) of the sample was born in the United States and most (76.5\%) spoke only or mostly the English language in their home. A high percentage of mothers and fathers graduated from high school (HS; $71.2 \%$ and $65.2 \%$ respectively). However, $18.5 \%$ and $24.7 \%$ of the students sampled did not know the highest level of education for their mother and father, respectively. Hispanic and non-Hispanic participants differed significantly in the following areas: urbanicity, birth country, language(s) spoken at home, and mother and father's level of education.

\section{Body Mass Index}

The average BMI among the Hispanic sample (21.7; $\mathrm{n}=2327$ ) was higher than the non-Hispanic sample (20.8; $\mathrm{n}=$ $10,177 ; \mathrm{p}<0.001)$. Although this finding was significant, homogeneity of variance was violated meaning the significance test lacks accuracy in interpretation, likely caused from the extreme difference in sample size of the groups. Nonetheless, the Hispanic sample also had higher rates of overweight and obesity for computed BMI-for-age categories $(p<0.001)$ compared to the non-Hispanic sample. The frequencies for computed BMI-for-age categories for Hispanic and non-Hispanic participants can be found in Table 2. 
Table 1: Characteristics of Entire, Hispanic, and Non-Hispanic Samples

\begin{tabular}{|c|c|c|c|c|c|}
\hline Question & Answer & $\begin{array}{l}\text { Entire Sample } \\
(\mathrm{n}=14,817 ; \%)\end{array}$ & $\begin{array}{c}\text { Hispanic } \\
(\mathrm{n}=\mathbf{2 , 8 8 9} ; \%)\end{array}$ & $\begin{array}{l}\text { Non-Hispanic } \\
(\mathrm{n}=11,559 ; \%)\end{array}$ & P Value $^{1}$ \\
\hline \multirow[t]{2}{*}{ Gender: } & Boy & 47.8 & 47.5 & 47.7 & 0.897 \\
\hline & Girl & 52.2 & 52.5 & 52.3 & \\
\hline Age: & & $\begin{array}{l}13.30 \pm 1.56 \\
(\text { Mean } \pm \text { SD) }\end{array}$ & $\begin{array}{l}13.32 \pm 1.55 \\
(\mathrm{Mean} \pm \mathrm{SD})\end{array}$ & $\begin{array}{l}13.32 \pm 1.58 \\
(\text { Mean } \pm \text { SD) }\end{array}$ & 0.934 \\
\hline \multirow[t]{3}{*}{ Urbanicity: } & Urban & 42.5 & 58.8 & 38.4 & $<0.01$ \\
\hline & Suburban & 28.6 & 22.9 & 30.2 & \\
\hline & Rural & 28.9 & 18.3 & 31.4 & \\
\hline \multirow[t]{2}{*}{ Birth Country: } & U.S.A. & 92.6 & 82.6 & 95.1 & $<0.01$ \\
\hline & Other & 7.4 & 17.4 & 4.9 & \\
\hline \multirow[t]{3}{*}{$\begin{array}{r}\text { Home } \\
\text { Language: }\end{array}$} & English & 76.5 & 27.4 & 88.5 & $<0.01$ \\
\hline & Other & 8.1 & 27.1 & 3.5 & \\
\hline & English \& Other & 15.4 & 45.5 & 8.0 & \\
\hline \multirow[t]{3}{*}{ EDU Mother: } & $\begin{array}{c}\text { Did not graduate } \\
\text { HS }\end{array}$ & 10.3 & 21.9 & 7.4 & $<0.01$ \\
\hline & $\begin{array}{c}\text { Graduated HS } \\
\text { but not College }\end{array}$ & 38.4 & 32.3 & 40.0 & \\
\hline & $\begin{array}{l}\text { Graduated } \\
\text { College }\end{array}$ & 32.9 & 19.4 & 36.4 & \\
\hline \multirow[t]{3}{*}{ EDU Father: } & Did not graduate & 10.1 & 20.8 & 7.6 & $<0.01$ \\
\hline & $\begin{array}{c}\text { Graduated HS } \\
\text { but not College }\end{array}$ & 34.7 & 30.5 & 35.9 & \\
\hline & $\begin{array}{l}\text { Graduated } \\
\text { College }\end{array}$ & 30.4 & 17.8 & 33.7 & \\
\hline
\end{tabular}

Table 2: BMI-for-age Categories by Ethnicity

\begin{tabular}{llcc}
\hline Percentiles & Interpretation & Non-Hispanic (\%) & Hispanic (\%) \\
\hline$<5^{\text {th }}$ & Underweight & 4.3 & 4.3 \\
\hline Between $5^{\text {th }}-85^{\text {th }}$ & Healthy Weight & 68.5 & 60.2 \\
\hline Between $85^{\text {th }}-95^{\text {th }}$ & Overweight & 15.9 & 20.1 \\
\hline$>95^{\text {th }}$ & Obese & 11.3 & 15.4 \\
\hline
\end{tabular}

Fruit and Vegetable Intake

There was a significant difference of fruit intake $(p<0.001)$ and vegetable intake $(p<0.001)$ between the Hispanic and non-Hispanic groups. A higher percentage of Hispanic students ate fruit once a week or less (35\%) compared to non-Hispanic students (30.5\%). Likewise, a lower percentage of Hispanic students ate fruits 5-6 days a week or more $(36.5 \%)$ compared to non-Hispanic students $(39.2 \%)$. Similarly, vegetable intake of once a week or less was $47.3 \%$ for Hispanics and $30 \%$ for non-Hispanics. Vegetable intake of 5-6 days a week or more was only $30.9 \%$ for Hispanics compared to $46.5 \%$ for non-Hispanics.

\section{Sugar-Sweetened Beverages}

A significant difference in soft drink consumption was found between Hispanic and non-Hispanic groups ( $p=0.001$ ), where Hispanic children drank coke or soft drinks more times per week. For Hispanic students, $60.3 \%$ reported soft drink consumption 5-6 days a week or more, compared to $58.3 \%$ of non-Hispanic students. Additionally, non-Hispanic students had a higher percentage of consumption reported once a week or less $(25.1 \%)$ compared to Hispanic students $(22.1 \%)$.
Physical activity

Survey results of physical activity in the past 7 days were significantly different between the Hispanic and non-Hispanic groups $(\mathrm{p}<0.001)$. Hispanic students reported being less physically active in the past 7 days, as $28.6 \%$ of students reported being physically active 2 days or less, compared to $24 \%$ of their non-Hispanic counterparts. Similarly, less Hispanic students reported being physically active in the past 5 days or more (48\%), compared to their non-Hispanic peers $(52.1 \%)$.

The amount of physical activity in a usual week also varied significantly between Hispanic and non-Hispanic students $(\mathrm{p}<0.001)$. Hispanic students reported typical physical activity of 2 days or less per week more often than non-Hispanic students (30\% and $23.6 \%$, respectively). Also, less Hispanic students reported typical physical activity of 5 days or more per week (45.5\%) than non-Hispanic students $(52.2 \%)$. 


\section{Discussion}

This secondary analysis used a nationally representative dataset of $6^{\text {th }}-10^{\text {th }}$ grade students from across the United States. The purpose of this analysis was to examine the differences in overweight/obesity and dietary and physical activity behaviors between Hispanic and non-Hispanic children living in the United States.

For descriptive factors, gender and age were not significantly different between the Hispanic and non-Hispanic groups. Significantly more Hispanic students lived in urban areas than non-Hispanic students. Significantly less Hispanic students spoke only or mostly English at home and were born in the United States compared to non-Hispanic students. The language spoken within the home and the birth country based on generational immigration are indicators of acculturation, where speaking mostly English in the home and being born in the United States are indicators of being more acculturated (Gordon-Larsen, Harris, Ward, \& Popkin, 2003; Lawton \& Gerdes, 2014). When Hispanic children living in the U.S but born in another country get more accustomed to American culture, negative changes in diet tend to occur (Greaney et al., 2012). This analysis did not sort Hispanic children by birth country to determine who had lower diet quality, but the majority $(82.6 \%)$ was born in the U.S., indicating a high level of acculturation. The results support a probable relationship between higher level of acculturation in this sample and poorer diet quality in Hispanic students.

Education level has been associated with obesity among adults in that college graduates have a lower rate of obesity $(21 \%)$ than those who only had some college-level education (29\%) and those who did not finish high school (33\%; Zoler, 2011). The weight status of the parent(s) may also impact the child's weight, with parent and child BMI z-scores being highly correlated (Saelens et al., 2012). Therefore, parental education level has an indirect influence on childhood obesity.

The results of this study supported the hypotheses. First, it was hypothesized that Hispanic children would have a higher rate of overweight and obesity than non-Hispanic children. The BMI-for-age categories between the Hispanic and non-Hispanic groups were found to be significantly different in that Hispanic students had higher rates of overweight and obesity. This finding coincides with previous research (Cunningham et al., 2014; Ogden et al., 2014; Zoler, 2011).

The behaviors affecting obesity examined within this analysis included fruit and vegetable intake, consumption of sugar-sweetened beverages, and physical activity. It was hypothesized that Hispanic children would have lower fruit and vegetable intake than non-Hispanic children. The results supported this hypothesis by indicating less intake of fruits and vegetables overall for Hispanic children compared to nonHispanic children. Additionally, it was hypothesized that Hispanic children would consume more sugar-sweetened beverages than non-Hispanic children. Self-reported consumption of sugar-sweetened beverages was significantly different with Hispanic children reporting soft drink consumption more often than non-Hispanic children. Lower consumption of fruits and vegetables and higher consumption of sugar-sweetened beverages has been linked with acculturation of Hispanic students (Dave et al., 2009; Wiley et al., 2014).

Lastly, it was hypothesized that Hispanic children would have lower levels of physical activity than non-Hispanic children. For physical activity in both the past 7 days and in a usual week, Hispanic children reported being physically active for fewer days compared to non-Hispanic children. Physical activity levels among children influences obesity prevalence in that higher levels of sport participation and outdoor play correlate with a decreased BMI, and higher levels of television and computer gaming correlate with an increased BMI (Katz, 2011; Wijtzes et al., 2014). Given the established relationship between these variables and obesity, it is plausible that these behaviors may contribute to the higher prevalence rates of overweight and obesity seen in Hispanic children.

While interpreting the results of this study, it is important to note the limitations. One limitation is the age of the data set. Due to time constraints, a more recent dataset could not be used for this analysis. Another limitation is the unequal sub-sample groups. There were several thousand more nonHispanic participants than Hispanic participants. Unequal sample sizes can skew variances and should be interpreted with caution when evaluating significance, but the percentage value of each group helps to verify the findings of this study. Furthermore, it is important to note that the non-Hispanic group also includes other minority groups and does not consist solely of non-Hispanic whites.

Further research on overweight and obesity in Hispanic children is recommended. It would be useful to look at similar variables for Hispanic students compared to other individual ethnic groups (e.g. African American or Caucasian) and also to compare the findings from this study to a more recent dataset in order to examine trends or changes over time. Additional research to determine Hispanic parent and child knowledge and practices regarding dietary recommendations would be interesting. Also, follow-up studies could help create culturally sensitive health promotion programs for Hispanic families.

In conclusion, Hispanic children as evidenced by the 2001-2002 Health Behavior of School-Aged Children Survey, have higher rates of overweight and obesity, are consuming less fruits and vegetables and more sugar-sweetened beverages, and have lower rates of physical activity compared to non-Hispanic children. These variations in dietary and physical activity behaviors are likely factors contributing to the increased prevalence of overweight and obesity in Hispanic children living in the United States.

\section{References}

Baskin, M. L., Ard, J., Franklin, F., \& Allison, D. B. (2005). Prevalence of obesity in the United States. Obesity Reviews, 6(1), 5-7. doi: 10.1111/j.1467-789X.2005.00165.x

Cason, K., Nieto-Montenegro, S., \& Chavez-Martinez, A. (2006). Food choices, food sufficiency practices, and nutrition education needs of Hispanic migrant workers in Pennsylvania. Topics in Clinical Nutrition, 21(2), 144-158. Cunningham, S. A. P., Kramer, M. R. P., \& Narayan, K. M. V. (2014). Incidence of childhood obesity in the United States. The New England Journal of Medicine, 370(5), 403411. 
Daniels, S. R. (2006). The consequences of childhood overweight and obesity. The Future of Children, 16(1), 4767. doi: $10.2307 / 3556550$

Dave, J. M., Evans, A. E., Saunders, R. P., Watkins, K. W., \& Pfeiffer, K. A. (2009). Associations among food insecurity, acculturation, demographic factors, and fruit and vegetable intake at home in Hispanic children. Journal of the American Dietetic Association, 109(4), 697-701. doi: http://dx.doi.org/10.1016/j.jada.2008.12.017

Gordon-Larsen, P., Harris, K. M., Ward, D. S., \& Popkin, B. M. (2003). Acculturation and overweight-related behaviors among Hispanic immigrants to the US: The national longitudinal study of adolescent health. Social Science \& Medicine, 57(11), 2023-2034.

Greaney, M. L., Lees, F. D., Lynch, B., Sebelia, L., \& Greene, G. W. (2012). Using focus groups to identify factors affecting healthful weight maintenance in Latino immigrants. Journal of Nutrition Education \& Behavior, 44(5), 448-453. doi: 10.1016/j.jneb.2011.11.008

Katz, D. L. (2011). Unfattening our children: Forks over feet. International Journal of Obesity, 35(1), 33-37. doi: 10.1038/ijo.2010.218

Lawton, K., \& Gerdes, A. (2014). Acculturation and Latino adolescent mental health: Integration of individual, environmental, and family influences. Clinical Child \& Family Psychology Review, 17(4), 385-398. doi: 10.1007/s10567-014-0168-0

Liu, J., Probst, J. C., Harun, N., Bennett, K. J., \& Torres, M. E. (2009). Acculturation, physical activity, and obesity among Hispanic adolescents. Ethnicity \& Health, 14(5), 509-525. doi: 10.1080/13557850902890209

Ogden, C. L., Carroll, M. D., Kit, B. K., \& Flegal, K. M. (2014). Prevalence of childhood and adult obesity in the United States, 2011-2012. JAMA: Journal of the American Medical Association, 311(8), 806-814. doi:

10.1001/jama.2014.732

Saelens, B. E., Sallis, J. F., Frank, L. D., Couch, S. C., Zhou, C., Colburn, T., ... Glanz, K. (2012). Obesogenic neighborhood environments, child and parent obesity: The neighborhood impact on kids study. American Journal of Preventive Medicine, 42(5), e57-e64. doi: 10.1016/j.amepre.2012.02.008

U.S. Dept. of Health and Human Services, Health Resources and Services Administration, Maternal and Child Health Bureau. (2003). Health behavior in school-aged children survey, 2001-02. Retrieved from: http://www.hbsc.org.

Wijtzes, A. I., Bouthoorn, S. H., Jansen, W., Franco, O. H., Hofman, A., Jaddoe, V. W. V., \& Raat, H. (2014).

Sedentary behaviors, physical activity behaviors, and body fat in 6-year-old children: The generation R study. International Journal of Behavioral Nutrition \& Physical Activity, 11(1), 1-18. doi: 10.1186/s12966-014-0096-x

Wiley, J. F., Cloutier, M. M., Wakefield, D. B., Hernandez, D. B., Grant, A., Beaulieu, A., \& Gorin, A. A. (2014). Acculturation determines BMI percentile and noncore food intake in Hispanic children 1-3. The Journal of Nutrition, 144(3), 305-310.

Zoler, M. L. (2011, 2011/02//). CDC: Obesity rates increased again in 2009. Clinical Psychiatry News, 39, 47. 